\title{
Aberration-Corrected Scanning Transmission Electron Microscopy and Energy- Dispersive Spectral Maps of DNA Origami Triangles Using Graphene Supports
}

\author{
Todd H. Brintlinger ${ }^{1}$, Nabil D. Bassim ${ }^{1}$, Rhonda M. Stroud ${ }^{1}$, Jeremy T. Robinson ${ }^{2}$, Susan Buckhout- \\ White $^{3}$, Mario Ancona ${ }^{2}$ and Ellen Goldman ${ }^{3}$ \\ 1. Materials Sci. \& Tech. Division, U.S. Naval Research Laboratory, Washington, DC. \\ 2. Electronics Sci. \& Tech. Division, U.S. Naval Research Laboratory, Washington, DC. \\ 3. Center for Biomolecular Sci. \& Eng., U.S. Naval Research Laboratory, Washington, DC.
}

Molecular assembly using DNA nanostructures has recently been an active area of research [1], esp. in the years since the invention of DNA origami [2]. While progress has been made in controlling these structures, the physical characterization of DNA nanostructures remains inadequate. Atomic force microscopy (AFM), with its ease of use and ability to work in a native aqueous environment, is the default imaging method. Scanning transmission electron microscopy (STEM), typically assisted by either heavy-atom staining or cryo-TEM, is an attractive alternative. Compared to AFM, STEM offers increased spatial resolution, compatibility with three-dimensional samples, and chemical specificity. Here, energy dispersive x-ray spectroscopy (EDS) allows for a complementary characterization of DNA structures, which can locate labels that are chemically but not structurally distinct, and as a diagnostic.

In previous TEM work on double-stranded DNA itself, some of the techniques employed stains [3], intensity-based contrast between the DNA and substrate [4], or electron energy loss spectroscopy (EELS) of light elements such as DNA-containing phosphorus [5] to distinguish the molecule. Obviously these methods can also be applied to structured DNA, though additive staining by heavy atoms degrades resolution and EELS is more beneficial with light elements. With this in mind, we look to directly image and chemically map unstained DNA structures using EDS, aided by decreased background signals by using graphene substrates [6,7]. While a near-ideal atomically-thin support, graphene does present imaging challenges related to cleanliness. To mitigate these challenges, we employ a variety of post-growth treatments [8]. We utilize a Nion aberration-corrected STEM probe (UltraSTEM200X) with a large-angle EDS detector (Bruker XFlash SDD detector), and for a model DNA nanostructure we use the DNA origami triangle devised by Rothemund [2].

We investigated many DNA origami preparation methods and graphene types and preparations, with results shown in Figs 1 and 2. For Fig. 1, single-layer graphene was fabricated in-house following standard copper-based growth recipes, transferred to holey carbon grids (Quantifoil ${ }^{\circledR} \mathrm{R} 2 / 1$ ), and briefly oxygen plasma-treated in a microwave plasma generator (PlasmaPreen) for 10 second before DNA triangles were deposited following gel-purification and buffer exchange to reduce the salt content. As evidence of the resolution, the rhombohedral holes in the corners of the DNA origami triangles can be seen in the high-angle annular dark-field image of Fig. 1a. Figure 1b shows the EDS chemical map obtained for phosphorus. Similarly, Fig. 1c gives the EDS map for iodine, whose presence is expected due to the GelRed, an iodine-containing intercalator, used for band visualization in the gel purification. A measurable trace is visible, co-located with the DNA and the phosphorus, though at much lower density than the phosphorous as expected. To further explore the combination of STEM-EDS with high$\mathrm{Z}$ elements in DNA scaffolds, we use a hydrogen-annealed graphene substrate in Fig. 2, where the $\mathrm{BaCl}_{2}$ salt, critical to the formation of the DNA, is associated with the DNA triangles. In Fig. 2a, we again show the HAADF of a (distorted) triangle. In Fig. 2b, while the barium ions only lightly bind to the 
DNA, they adhere strongly enough to the DNA to be measurable via STEM-EDS. Lastly, we again show the EDS map of the iodine L- $\alpha$ lines. Here the total number of counts trail those for barium and phosphorus (not shown), but also seem co-located with the DNA scaffold. Our other DNA and graphene treatments will be discussed, along with the possibility of imaging individual high- $Z$ atoms within a DNA scaffold.

References:

[1] N Seeman in "Structural DNA Nanotechnology" (Cambridge Univ. Press, 2016).

[2] PWK Rothemund, Nature 440 (2006), p. 297.

[3] M Tanaka et al, Ultramicroscopy 1 (1975), p. 7.

[4] DC Bell et al, Microsc. Microanal. 18 (2007), p. 1049.

[5] M Aronova et al, Ultramicroscopy 107 (2007), p. 232.

[6] S Buckhout-White et al, SoftMatter 9 (2013), p. 1414.

[7] Y Kabiri et al, Small (2017), p. 1700876.

[8] T Brintlinger et al, Microsco. Microanal. 23(S1) (2017), p. 1740.
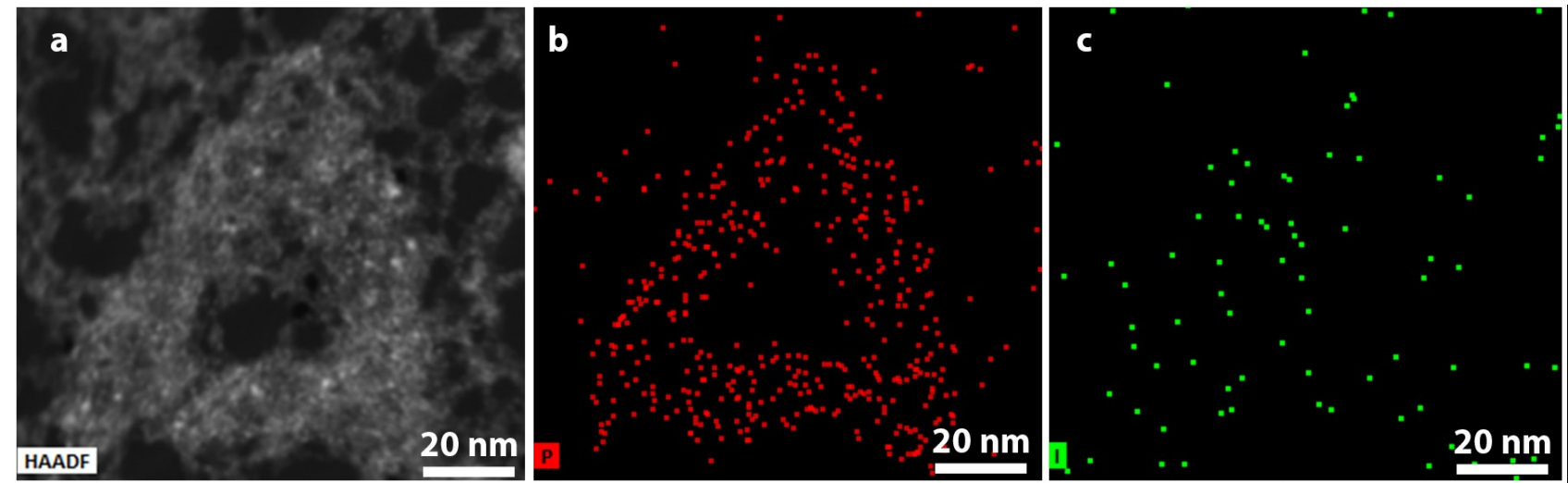

Figure 1. Aberration-corrected scanning transmission electron microscopy (STEM) and energy dispersive spectroscopy (EDS) of DNA origami triangle on plasma-treated graphene: (a) High-angle annular dark-field (HAADF) micrograph of unstained DNA on graphene at room temperature, (b) EDS map of phosphorous at L- $\alpha$ energy, and (c) EDS map of iodine L- $\alpha$.
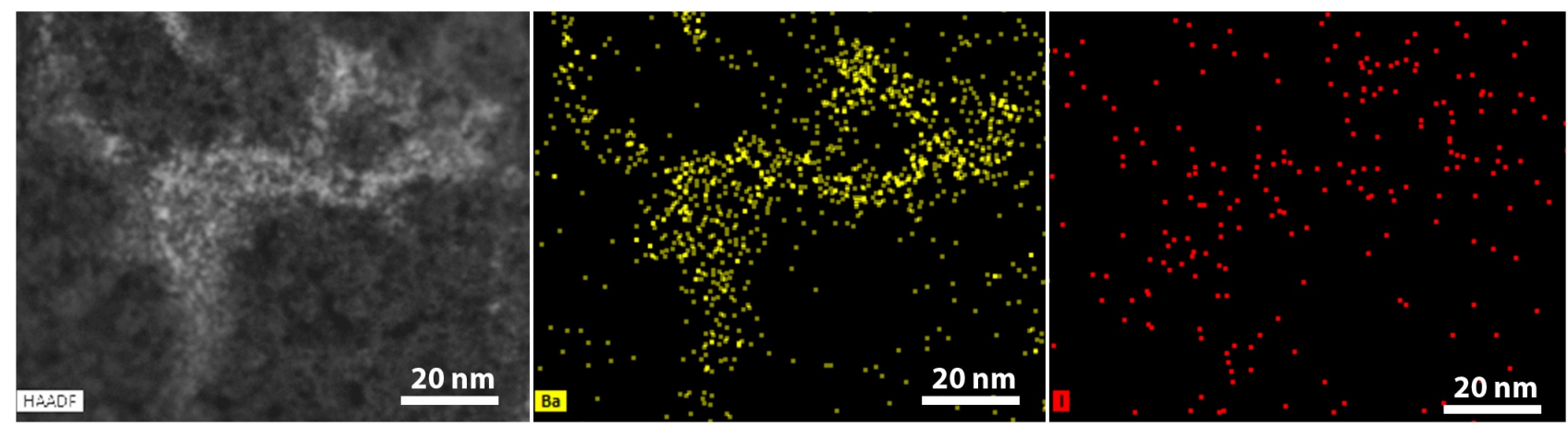

Figure 2. Aberration-corrected scanning transmission electron microscopy (STEM) and energy dispersive spectroscopy (EDS) of DNA origami triangle on hydrogen-annealed graphene: (a) High-angle annular dark-field (HAADF) micrograph of unstained DNA on graphene at room temperature, (b) EDS map of barium at L- $\alpha$ energy, and (c) EDS map of iodine L- $\alpha$. 